\title{
Flexible electroluminescent panels
}

\author{
V.I. Vlaskin ${ }^{1}$, S.I. Vlaskina ${ }^{2,3}$, O.Yu. Koval ${ }^{4}$, V.E. Rodionov ${ }^{2}$, G.S. Svechnikov ${ }^{2}$ \\ ${ }^{1} 11$, Rainbow Falls, Irvine, California 92603, USA \\ ${ }^{2}$ V. Lashkaryov Institute of Semiconductor Physics, NAS of Ukraine, 45, prospect Nauky, 03028 Kyiv, Ukraine \\ Phone 380(44) 525-37-92, fax 380 (44) 525-83-42; e-mail: businkaa@mail.ru \\ ${ }^{3}$ Dong Seoul College, 461-714, 423 \\ Bokjung-Dong, Sungnam-city, Kyonggi-do, Korea \\ Phone 82 (031) 7202141, fax 82(031) 7202261; e-mail: svitlana@haksan.dsc.ac.kr \\ ${ }^{4}$ Institute for Problems of Material Science, NAS of Ukraine, 3, Krzhizhanovsky str., 03142 Kyiv, Ukraine
}

\begin{abstract}
Recently developed ac ZnS-powder electroluminescence (EL) devices have flexibility (thickness is about $60 \mu \mathrm{m}$ ) and can be multisegment, multicolor, as well as rolled and bent. All colors (white, blue, blue-green, green, and orange) have been investigated for improvement of their operational characteristics. Many factors including the type of phosphor, formation method, contacts, initial input power (peak voltage and frequency), type of input power, and environment safety which define the panel's properties are discussed.
\end{abstract}

Keywords: electroluminescence, thick film display, powder, phosphor, flexibility.

Manuscript received 09.02.07; accepted for publication 24.04.07; published online 19.10.07.

\section{Introduction}

Effect of a non-thermal generation of light (electroluminescence) resulting from the application of an alternative electrical field to a $\mathrm{ZnS}$ powder was discovered in 1936 by the French physicist G. Destriau. The flexible electroluminescence panel (FELP) is essentially a capacitor structure with the powder of phosphor dielectric sandwiched between two electrodes, one of which is transparent to allow light to escape. Application of a voltage across the electrodes generates a changing electric field of the order of $10^{8} \mathrm{~V} / \mathrm{m}$ within the phosphor powder, which causes phosphor to emit light. Light is generated by the impact excitation of a light-emitting center, called an activator or a luminescent center, by high-energy electrons.

Phosphor powders are materials such as zinc sulfide, $\mathrm{ZnS}$, doped with $\mathrm{Cu}, \mathrm{Mn}, \mathrm{Er}, \mathrm{Tb}, \mathrm{Ga}$ etc. to achieve the emission colors. The color can be defined by the concentration of a doped metal during the manufacture of phosphor or can be achieved by blending phosphor powders of different colors to achieve composite color (blue-green, yellow-green, etc. White color is achieved either by using phosphor blends or by the optical shifting of the emission color.). ZnS powders are coated with metal oxides such as $\mathrm{Al}_{2} \mathrm{O}_{3}$ or $\mathrm{TiO}_{2}$. Depending on the particular application, FELPs use different kinds of polymer binders. The binders are often fluorine substituted polymers. New flexible EL materials [2] can be used for flexible panels with extremely low power draw and generate no heat under normal operating conditions. Using these materials make FELPs very flexible, very thin $(\sim 60 \mu \mathrm{m})$, and very soft. FELPs can bend to conform a product shape and operate while being flexed. Now they are used as backlights for key pad of cell phones.

FELPs have advantages over other EL panels that have a very thin thickness and lower cost. The particle size of the phosphor powder $(\sim 20 \mu \mathrm{m})$ provides the highest efficiency of light emission. Also, FELPs can replace the current methods of printing an embossed panel backed by a PC board with LED lights. They are so thin and flexible that they allow for downsizing the products. Other benefits include the imperviousness to shocks and vibration (for aerospace applications), negligible heat emission and electromagnetic interference (EMI), no radio frequency interference (RFI) (for aerospace applications), compatibility with night goggle vision. Moreover, the light angle can be controlled for military map reading applications. A uniform surface illumination of complex or flat shape, thinness, flexibility, low weight, low power consumption, very low heat generation, the environment safety, the voltage/frequency conditions, the possibility to deposit the light emitting particles on any surface, any design, or lettering production make FELPs very perspective for the everyday use. FELPs can be rolled, bent, and crushed and will normally continue to function (of course, sharp objects must be avoided). 
1

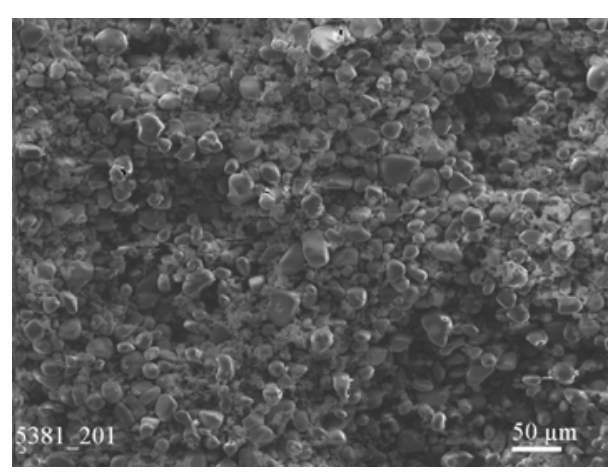

$\times 200$, SEI

4

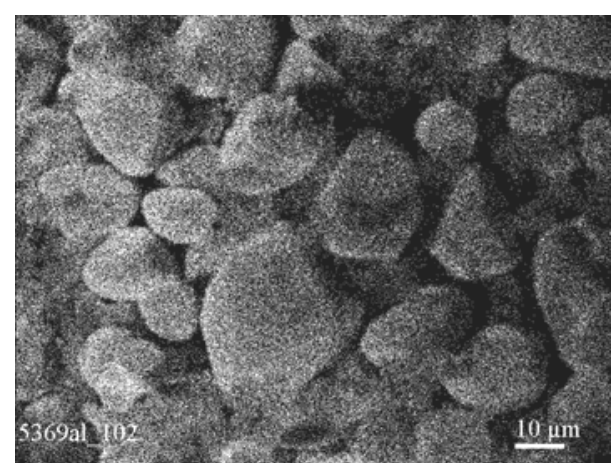

$\times 1000, X$-ray Al-K ${ }_{\alpha}$

5

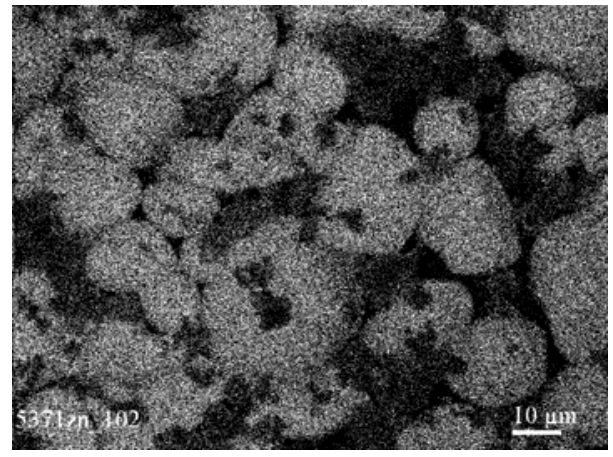

$\times 1000, X$-ray Zn-K ${ }_{\alpha}$

7

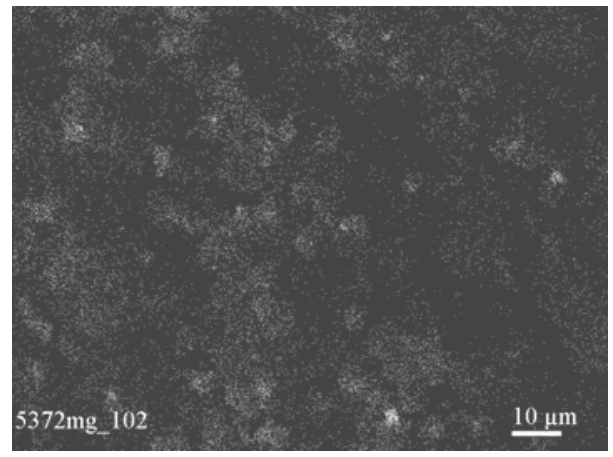

$\times 1000$, X-ray $\mathrm{Mg}_{\alpha}$
2

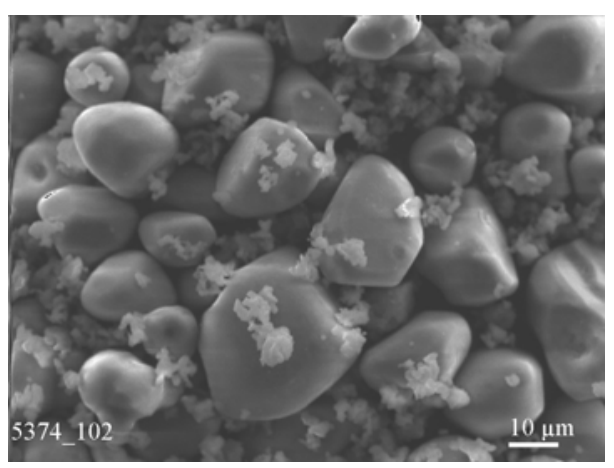

$\times 1000$, SEI

3

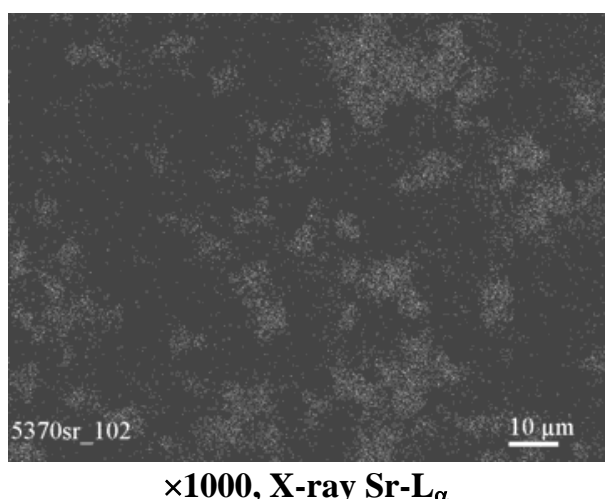

6

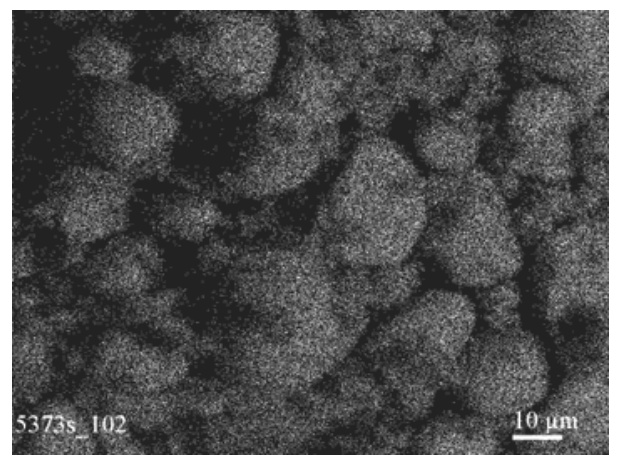

$\times 1000, X$-ray S-K ${ }_{\alpha}$ 
1

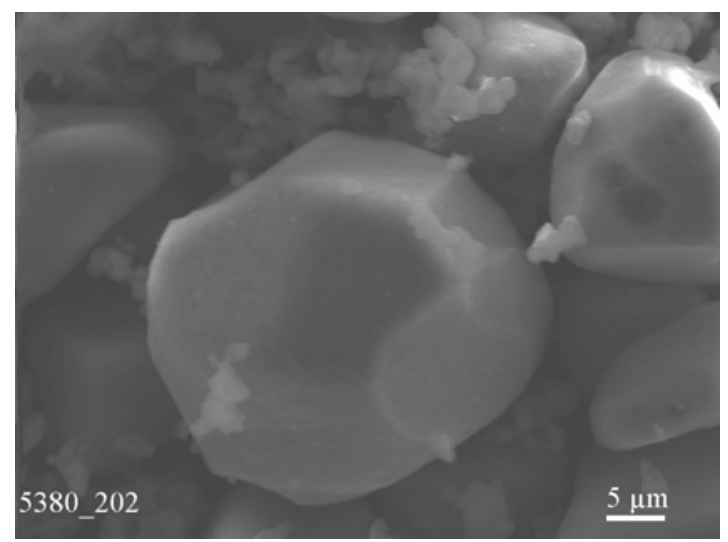

$\times 2000$, SEI

4

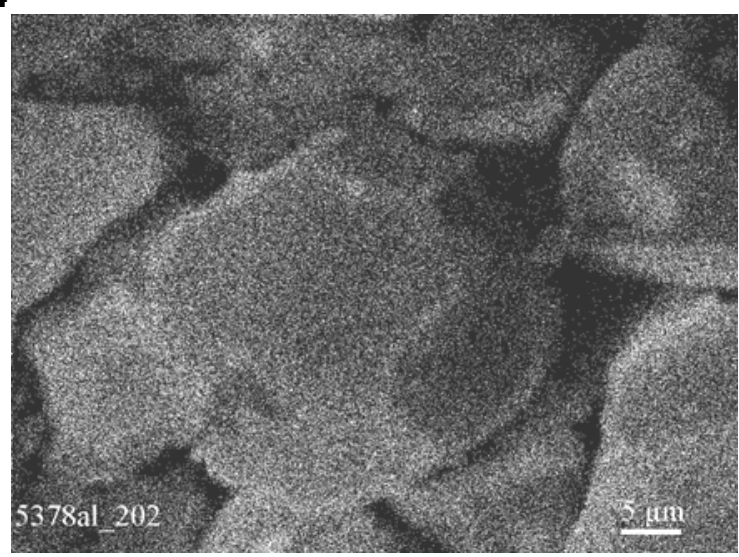

$\times 2000$, X-ray Al-K $\alpha$

6

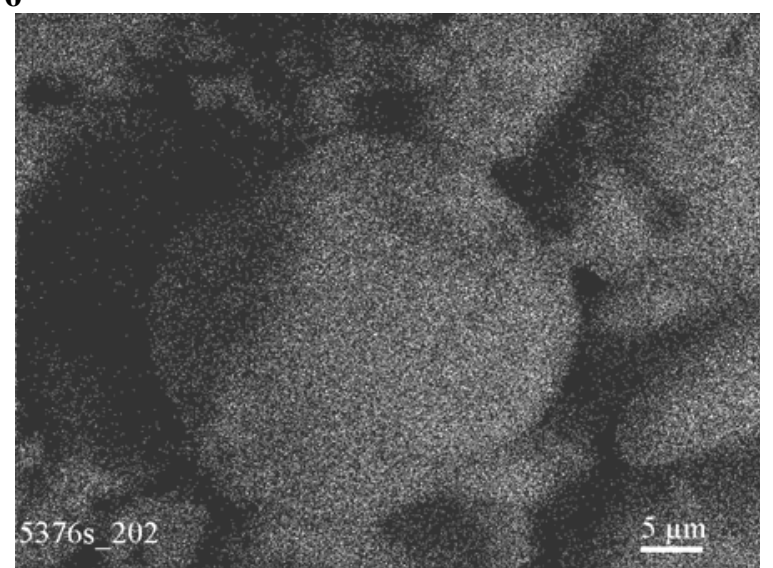

$\times 2000$, X-ray S-K
3

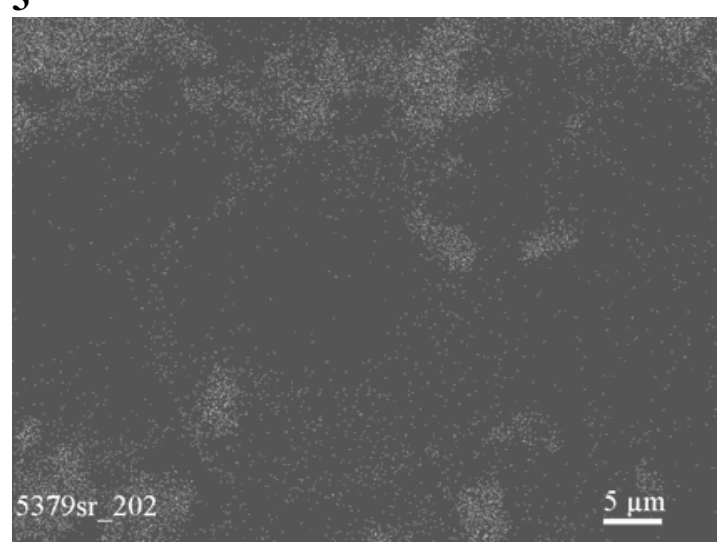

$\times 2000$, X-ray Sr- $\mathrm{L}_{\alpha}$

5

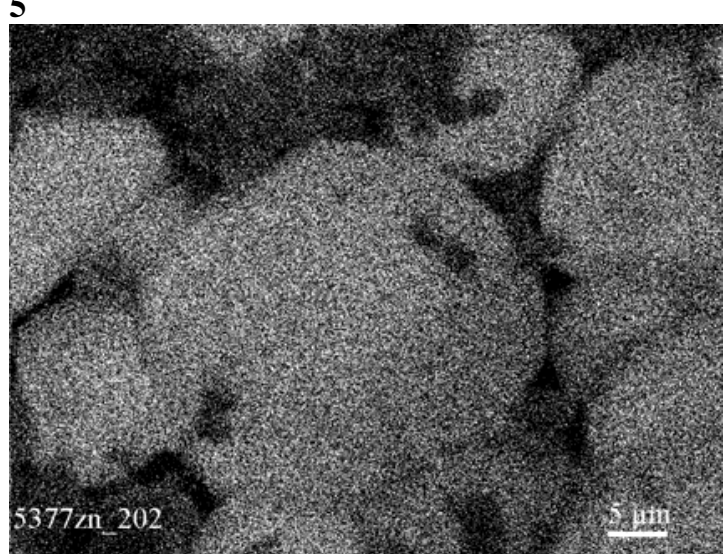

$\times 2000$, X-ray Zn-K $\alpha$

7

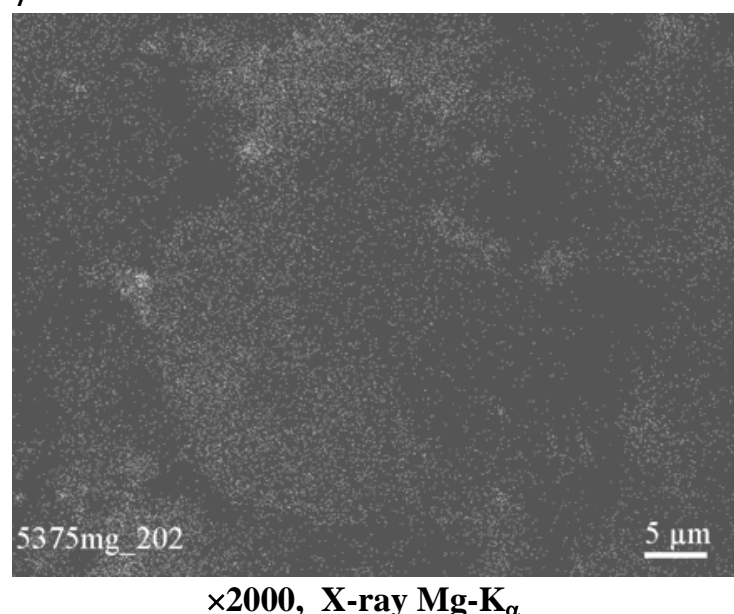

Fig. 2. Structure and distributions of chemical elements in FELPs (SEM, XRMA); ×2000. 
This article will focus on the investigation of some properties of flexible panels and on the discussion of some EL emission mechanism acting in flexible panels.

\section{Experiment}

Flexible EL panels had been prepared by the method of print technology [1, 2]. The fabrication of a FELP includes a non-adhesive shield polymer layer with thickness of $10-15 \mu \mathrm{m}$, one of conductive electrodes (1$10 \mu \mathrm{m})$, a dielectric layer $(5-10 \mu \mathrm{m})$, first field polymer layer $(0.5-1 \mu \mathrm{m})$, white phosphor layer $(20-25 \mu \mathrm{m})$, second field polymer layer $(0.5-1 \mu \mathrm{m})$, transparent electrode layer $(0.5-1 \mu \mathrm{m})$, and second shield polymer layer $(10-15 \mu \mathrm{m})$. The total thickness of FELP is about $60 \mu \mathrm{m}$. The powder of phosphor was provided by Sylvania Corp.

The preferred method is the screen-printing method that is a process, in which a layer is allowed to pass through the mesh made of silk or stainless steel, thereby forming a uniform layer. The thickness of a FELP is controlled by using the different meshes of screens and viscosities of binders.

Above the threshold voltage, electrons are injected from the interface states between the phosphor powder grains and insulating layers by high-field-assisted tunneling. Insulating layers are around of the every grain and cover all EL powder layers. Color of emission is controlled by the addition of different luminescent centers. Red color is based on luminescence of $\mathrm{ZnS}: \mathrm{Mn}$ or on SrS:CaS phosphor [3]. Green color is based on $\mathrm{ZnS}: \mathrm{Cu}, \mathrm{Al}$, or CdS. Blue color is based on $\mathrm{ZnS}: \mathrm{Ag}(\mathrm{Au})$ or ZnS/SrS:Ce (Ho) (or another lanthanides: Ce, Tm). The structure of white light-emitting powder obtained by secondary electron imagination (SEM) and X-ray microanalysis (XRMA) is shown in Figs. 1-3.

According to the microphoto of the active white EL layer, two kinds of phosphor powders are observed (Fig. 1). One of the types of the phosphor powder has the size of particles less than $26 \mu \mathrm{m}$, the second one less than $5 \mu \mathrm{m}$ (Figs. 2 and 3). XRMA in $\mathrm{Zn}-\mathrm{K}_{\alpha}$ and S$\mathrm{K}_{\alpha}$ gives evidence that the material of large particles of the powder is EL phosphor ZnS (Fig. 1, pictures 5, 6 at a magnification of 1000). The particles are coated by $\mathrm{Al}$ oxide (Fig. 2, picture 4). All images of ZnS powder in $\mathrm{X}$-ray $\mathrm{Mg}-\mathrm{K}_{\alpha}$ shows the presence of Mg (Fig. 2, picture $3,6)$.

Cathodoluminescent optical photo at a magnification of 400 (Fig. 4) under excitation by a $25-\mathrm{kV}$ electron beam gives evidence of two different photoluminescent materials that cause two different kinds of photo-luminescence - blue and red ones.

Also "blue-cathodoluminescence" large grains are coated by Mg (Fig. 2, picture 7). As usual, Mg gives the red color in the luminescent spectrum (Figs. 1 and 2). It is clear from XRMA-Mg that Mg coated ZnS grains (Figs. 1 and 2), namely, the magnesium atoms are in contact with the surface of $\mathrm{ZnS}$ grains. Due to the fact that $\mathrm{Mg}$ atoms diffuse into $\mathrm{ZnS}$ grains, red emitted color is present in photoluminescence.

The small-size powder (less than $5 \mu \mathrm{m}$ as seen in Fig. 3) reveals red cathodoluminescence (Fig. 4). The particles have another chemical structure as compared with large-size powder ZnS. The particles consists of strontium sulfide SrS (Fig. 2, picture 3,6). The mixture of two different, ZnS and SrS, electroluminescent materials provides white color of FELPs.

The electrons injected by voltage are accelerated in grains and gain the kinetic energy enough to excite luminescent centers or the host lattice. High-energy electrons (hot electrons) excite luminescent centers through the impact excitation mechanism. When electrons in the excited states of luminescent centers make radiative transition to the ground states, electroluminescent emission in $\mathrm{ZnS}$ grains is realized [4, 5]. The blue emission of $\mathrm{ZnS}$ is absorbed by SrS red color emission phosphor grains. In the case of SrS grains, the additional mechanism takes place and is characterized by the field-induced ionization of luminescent centers and the subsequent trapping of lowenergy electrons, resulting in EL emission [5].

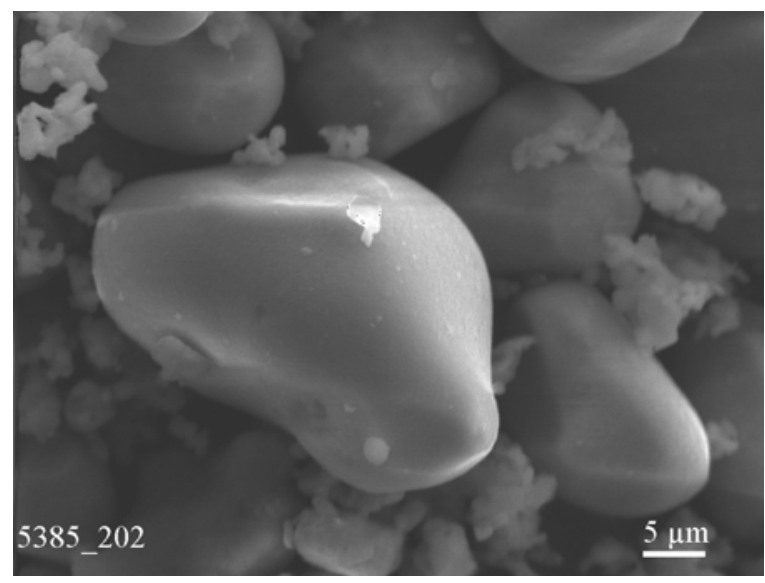

Fig. 3. Microphoto of a powder in FELP; $\times 2000$.

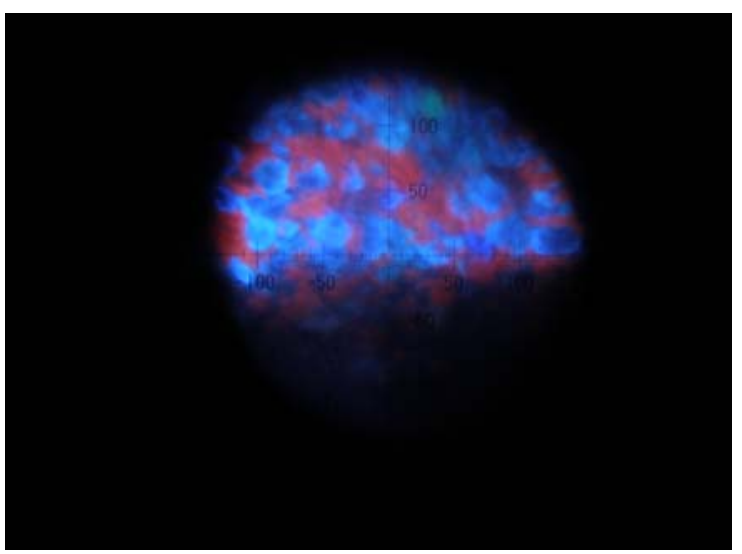

Fig. 4. Cathodoluminescence of the FELP powder. 


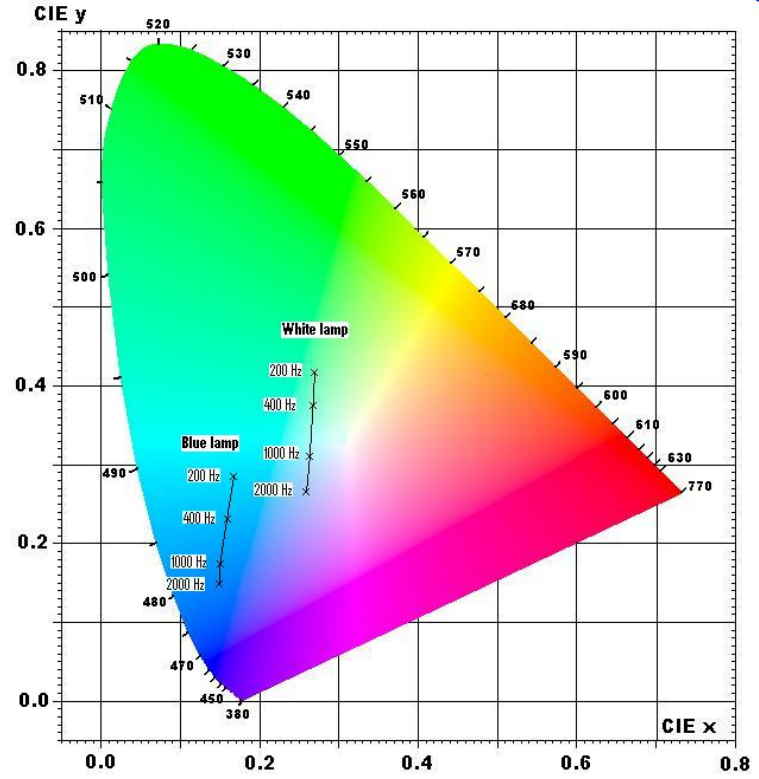

Fig. 5. Chromaticity diagram of two types of FELPs (blue and white).

The chromaticity diagram of two types of FELPs (blue and white) is shown in Fig. 5.

A color is shifted to blue as the frequency increases. Namely, blue color dominates at higher frequencies because of the less relaxation time for bluecolor-responsible centers as compared with green-colorresponsible luminescence centers [4]. When the green component is on the saturation, the blue component's intensity grows and will be on the saturation at higher frequencies (at least to $2000 \mathrm{~Hz}$ ). At the same frequency, increasing the voltage leads to the shift of color from green to blue just for the same reason: the saturation for blue-centers occurs at a higher voltage than for greencenters because of different time constants [4].

\section{Conclusion}

We have investigated the characteristics of FELPs (in particular, those of white and blue FELPs) and the influence of surface processing, formation method, and doping with luminescence materials on the properties of FELPs. White FELPs can be used for flexible panels with extremely low power draw. It is shown that the FELP-based structures possess a high stability of light emission.

\section{References}

1. V. Vlaskin, AC powder electroluminescence device and method for making the same. USA Patent 5,912,533, June 15, 1999.

2. V. Vlaskin, Flexible electroluminescent material. USA Patent 7,148,623, December 12, 2006.

3. Tian et al., Red photoluminescent phosphors. USA Patent 6,783,700, August 31, 2004.

4. H.K. Henish, Electroluminescence. Pergamon Press, New York, 1962.

5. Y.A. Ono, Electroluminescent displays. World Scientific, Singapore, 1995. 